\title{
Response to editorial comment "A retrosigmoid ileal conduit might prevent ureteroileal anastomotic stricture after ileal conduit diversion"
}

\author{
Gianluca Giannarini ${ }^{1}$, Alessandro Crestani ${ }^{1}$, Vincenzo Ficarra ${ }^{2}$ \\ ${ }^{1}$ Urology Unit, Academic Medical Centre Hospital "Santa Maria della Misericordia”, Udine, Italy; ${ }^{2}$ Department of Human and Paediatric Pathology \\ "Gaetano Barresi”, Urologic Section, University of Messina, Messina, Italy \\ Correspondence to: Prof. Vincenzo Ficarra, MD, FEBU. Department of Human and Paediatric Pathology "Gaetano Barresi”, Urologic Section, \\ University of Messina, via Consolare Valeria 1, 98124 Messina, Italy. Email: vficarra@unime.it. \\ Provenance: This is an invited article commissioned by the Section Editor Xiao Li (Department of Urology, Jiangsu Cancer Hospital \& Jiangsu \\ Institute of Cancer Research \& Nanjing Medical University Affiliated Cancer Hospital, Nanjing, China). \\ Response to: Gomes CM, Laferreira LS. A retrosigmoid ileal conduit might prevent ureteroileal anastomotic stricture after ileal conduit diversion. \\ Transl Androl Urol 2018. doi: 10.21037/tau.2018.10.10
}

Submitted Nov 09, 2018. Accepted for publication Nov 20, 2018.

doi: $10.21037 /$ tau. 2018.11 .08

View this article at: http://dx.doi.org/10.21037/tau.2018.11.08

We thank the authors for their interest in our recent article describing the surgical technique and early functional results of a retrosigmoid ileal conduit diversion after radical cystectomy (RC) (1). In a single-centre single-surgeon comparative study with a short-term follow-up, we observed a significantly reduced rate of ureteroileal anastomotic stricture (UAS) and no increase in intra- or postoperative complications with the retrosigmoid versus traditional Wallace ileal conduit. In details, no cases of UAS were detected in 30 patients who had received a retrosigmoid ileal conduit with a direct end-to-side ureteroileal anastomosis after a mean follow-up time of 10.8 months.

Following these encouraging results, the retrosigmoid approach has been applied to all patients receiving RC with ileal conduit at our institutions. Between April 2017, i.e., the termination date of the above comparative study, and September 2018 a further 33 patients treated with $\mathrm{RC}$ received a retrosigmoid ileal conduit, thus resulting in a prospective cohort of 63 patients. The latter patients were treated at two academic centres by one of three surgeons with varying experience. After a mean followup of 18.2 months there were still no cases of UAS. No intraoperative complications were observed. Ninety-day overall complications, classified according to the Dindo modification of the Clavien system (2), were recorded in 28/63 (44\%) patients, and major complications in 12/63 (19\%) patients. Most common major complications were represented by wound infection/dehiscence and lymphocele. No single case of ureteroileal anastomotic leakage or any urinary fistula was observed.

We would like to address the authors' concern on the applicability of this technique to obese patients by noting that $16 / 63(25 \%)$ patients in our series had a body mass index exceeding $30 \mathrm{~kg} / \mathrm{m}^{2}$. The retrosigmoid approach was feasible in all of them with no increase in operating room time or morbidity. The length of the ileal segment that has to be taken to build the conduit can easily be adapted to the size and thickness of the mesentery and sigmoid.

We would also like to mention a potential condition where the retrosigmoid ileal conduit would be useful. If a patient has a high risk of experiencing unilateral nodal progression (i.e., bulky regional nodal disease, incomplete regional lymph node dissection), then a traditional conduit diversion with both ureters on the same side of the pelvis/ retroperitoneum would predispose him to bilateral ureteric obstruction in case of nodal compression/infiltration occurring in the ipsilateral side. On the contrary, if the ureters are kept in their naïve location using a retrosigmoid 
approach for the ileal conduit, one renal unit remains safe in case of unilateral nodal progression.

The retrosigmoid approach has now become the standard option for patients receiving an ileal conduit in our clinical practice. We will continue to follow-up our patients in order to assess the long-term functional results, although it is known that the risk of UAS is very low after 18 months after surgery, as already acknowledged by the authors. Our data await external validation from large cohorts of other centres. We also look forward to studies where a robotassisted approach for this technique is attempted.

\section{Acknowledgements}

None.

Cite this article as: Giannarini G, Crestani A, Ficarra V. Response to editorial comment "A retrosigmoid ileal conduit might prevent ureteroileal anastomotic stricture after ileal conduit diversion". Transl Androl Urol 2018;7(Suppl 6):S768S769. doi: $10.21037 /$ tau.2018.11.08

\section{Footnote}

Conflicts of Interest: The authors have no conflicts of interest to declare.

\section{References}

1. Ficarra V, Giannarini G, Crestani A, et al. Retrosigmoid Versus Traditional Ileal Conduit for Urinary Diversion After Radical Cystectomy. Eur Urol 2018. [Epub ahead of print].

2. Dindo D, Demartines N, Clavien PA. Classification of surgical complications: a new proposal with evaluation in a cohort of 6336 patients and results of a survey. Ann Surg 2004;240:205-13. 